\title{
Direct measurement of the intrinsic electric dipole moment in pear-shaped thorium-228
}

\author{
M. M. R. Chishti, ${ }^{1, *}$ D. O’Donnell,,${ }^{1, \dagger}$ G. Battaglia, ${ }^{2}$ M. Bowry,${ }^{1}$ D. A. Jaroszynski, ${ }^{2}$ \\ B. S. Nara Singh, ${ }^{1}$ M. Scheck, ${ }^{1}$ P. Spagnoletti, ${ }^{1}$ and J. F. Smith ${ }^{1}$ \\ ${ }^{1}$ SUPA, School of Computing, Engineering and Physical Sciences, \\ University of the West of Scotland, \\ Paisley, PA1 2BE, United Kingdom. \\ ${ }^{2} S U P A$, Department of Physics, University of Strathclyde, \\ Glasgow, G4 ONG, United Kingdom.
}

(Dated: June 9, 2020)

\begin{abstract}
Atomic nuclei with certain combinations of proton and neutron numbers can adopt reflectionasymmetric or octupole-deformed shapes at low excitation energy. These nuclei present a promising avenue in the search for a permanent atomic electric dipole moment the existence of which has implications for physics beyond the Standard Model of particle physics. Theoretical studies have suggested that certain thorium isotopes may have large octupole deformation. However, due to experimental challenges, the extent of the octupole collectivity in the low-energy states in these thorium nuclei has not yet been demonstrated. Here, we report measurements of the lifetimes of low-energy states in ${ }^{228} \mathrm{Th}(Z=90)$ with a direct electronic fast-timing technique, the mirror symmetric centroid difference method. From lifetime measurements of the low-lying $J^{\pi}=1^{-}$and $J^{\pi}=3^{-}$states, the $E 1$ transition probability rates and the intrinsic dipole moment are determined. The results are in agreement with those of previous theoretical calculations, allowing us to estimate the extent of the octupole deformation of ${ }^{228} \mathrm{Th}$. This study indicates that the nuclei ${ }^{229} \mathrm{Th}$ and ${ }^{229} \mathrm{~Pa}(Z=91)$ may be good candidates for the search for a permanent atomic electric dipole moment.
\end{abstract}

\footnotetext{
*Department of Physics, University of Surrey, Guildford, GU2 7XH, United Kingdom.

${ }^{\dagger}$ Electronic address: david.odonnell@uws.ac.uk
} 
Bohr and Mottelson interpreted observed spectra of deformed atomic nuclei as the manifestation of rotational and vibrational degrees of freedom $[1,2]$. In this pioneering work, the excitations were understood as rotational or vibrational modes of quadrupole deformed axially-symmetric nuclei. Since then it has became clear that some nuclei may also adopt reflection asymmetric shapes, such as those resulting from long-range octupole-octupole residual interactions. The operator associated with such interactions has negative parity, which leads to the nucleus adopting shapes where reflection symmetry is broken. The distinctive shape associated with static octupole deformation has led to such nuclei being referred to as pear shaped, where the nuclear density is higher at one pole than the other. Such octupole deformation can result in a separation of the centres of charge and mass which can result in a sizeable electric dipole $(E 1)$ moment, which will be observable through enhanced $E 1$ transitions between nuclear states [3].

Reflection asymmetric nuclei have attracted attention in recent years in the search for a permanent atomic electric dipole moment (EDM). The Standard Model predicts a vanishingly small, but non-zero, EDM. To date, no evidence has been found that supports this prediction, despite several experiments having been undertaken using different methods [48]. This is because the EDM predicted by the Standard Model is far below the sensitivity of current experimental capabilities. Nevertheless, by placing upper limits on an EDM, these studies have successfully constrained any proposed extensions of the Standard Model predicting a significantly larger EDM value. The ACME II experiment, which has recently improved on the upper limit on the EDM value by an order of magnitude [8], involves laser spectroscopy of thorium monoxide molecules to measure the EDM. Some isotopes of thorium are predicted to exhibit an enhanced octupole collectivity at low excitation energies [9]. The use of such nuclei in the search for an EDM would be particularly attractive because the induced E1 moment resulting from the reflection asymmetric shape enhances the nuclear Schiff moment [10-13], and therefore the atomic EDM, by two to three orders of magnitude $[14,15]$. In particular, Ref. [14] shows that the Schiff moment in the laboratory frame exhibits a quadratic dependence on the extent of the octupole deformation.

One nucleus identified early as an ideal candidate with which to search for an EDM is ${ }^{229} \mathrm{~Pa}$ $(Z=91)$. Possessing an odd number of protons, this nucleus was predicted to have a lowlying parity doublet in which two states with the same total angular momentum are found almost degenerate in energy [16]. Such a doublet, which is regarded as a signature of octupole 
correlations, was first reported by Ahmad et al. [17] in 1982. More recently, however, doubt has been cast upon the existence of this doublet due to a lack of conclusive evidence [18-20]. Should ${ }^{229} \mathrm{~Pa}$ indeed be octupole deformed in the ground state it is expected that the eveneven nucleus ${ }^{228} \mathrm{Th}(Z=90)$, which constitutes the core of ${ }^{229} \mathrm{~Pa}$ to which an unpaired proton is coupled, would also exhibit characteristics consistent with enhanced octupole correlations. Another nucleus where an enhanced nuclear Schiff moment is predicted is thorium-229, which corresponds to an unpaired neutron coupled to a ${ }^{228} \mathrm{Th}$ core [21]. This further emphasises the importance of determining the octupole deformation in ${ }^{228} \mathrm{Th}$.

A signature of reflection asymmetry in even-even nuclei is the presence of low-lying negative parity states. Such states, with total angular momentum values corresponding to $1 \hbar$ and $3 \hbar$, were first identified in $\alpha$-decay spectroscopy measurements in the 1950s $[22,23]$. Electromagnetic transitions between these negative parity states and members of the ground state band are also good indications of octupole collectivity. For instance, the observation of enhanced electric octupole (E3) transitions between the low-lying $J=3^{-}$state and the $J=0^{+}$ground state is considered as an unambiguous signature of octupole collectivity [24]. To date only two known nuclei have been found to exhibit both signatures: ${ }^{224} \mathrm{Ra}$ [25] and ${ }^{226} \mathrm{Ra}$ [26]. Nevertheless, there are a several actinide nuclei that have low-lying negative parity states; theoretical studies have suggested they should have appreciable octupole deformations in the ground state [9, 27]. Indeed, density functional theories used in Ref. [9] suggest that thorium nuclei are likely to have the largest octupole deformations of all the actinides. However, disagreement exist between different theoretical models on which thorium nucleus should exhibit the largest octupole collectivity. In the work of Agbemava et al. [9], pronounced quadrupole and octupole deformations are expected in the ground states of ${ }^{226} \mathrm{Th}$ and ${ }^{228} \mathrm{Th}$ and the octupole deformation parameter $\beta_{3}$ is maximised for the latter. In this paper we investigate the extent of octupole collectivity in ${ }^{228} \mathrm{Th}$ by measuring the enhancement of electric dipole transitions from the low-lying $J=1^{-}$and $3^{-}$states.

The observation of enhanced $E 1$ transitions connecting negative and positive parity states is generally considered a good indication of reflection asymmetry, albeit with some ambiguity $[3,28]$. One source of ambiguity arises from the enhancement of electric dipole transitions that are not unique to octupole deformed nuclei but also manifest in well-deformed reflectionsymmetric nuclei due to the presence of low-lying octupole vibrational states [28]. This is further complicated because the microscopic shell contribution to the dipole moment in the 
intrinsic frame effectively negates the macroscopic contribution from the reflection asymmetric shape of the nucleus, which was first identified by Butler and Nazarewicz [3]. The most striking example of this is observed in the work of Gaffney et al. [25] where they reported measurements of a large E3 strength in ${ }^{224}$ Ra. This indicates a sizeable octupole collectivity in the ground state, yet a small $E 1$ strength, consistent with reflection-symmetric nuclei, was also reported.

In our study we measure the lifetimes of low-lying excited states of ${ }^{228} \mathrm{Th}$ using electronic fast timing techniques based on fast scintillating $\gamma$-ray detectors. This represents the first measurement of the absolute electric dipole transition rates in any thorium isotopes, for the low-lying negative-parity states characteristic of octupole collectivity. When compared with calculations available in the literature [24] we conclude that the low-energy structure of ${ }^{228} \mathrm{Th}$ is consistent with a strongly quadrupole-deformed nucleus with an octupole deformation comparable with that reported in ${ }^{224} \mathrm{Ra}$ [25]. Our findings suggest that the odd- $A$ nuclei ${ }^{229} \mathrm{Th}$ and ${ }^{229} \mathrm{~Pa}$ may be good candidates with which to search for a permanent EDM.

Spectra of $\gamma$ radiation detected with the Start $\operatorname{LaBr}_{3}(\mathrm{Ce})$ and HPGe detectors are shown in Figure 1. The definition of the detectors can be found in the Methods section of this paper. Since data are only recorded when a TAC pulse is generated, the $\mathrm{LaBr}_{3}(\mathrm{Ce})$ spectrum of Figure 1 represents the projection of a two-dimensional $\gamma-\gamma$ (or Start-Stop) coincidence matrix. The HPGe spectrum, however, is the projection of a three-dimensional $\gamma-\gamma-\gamma$ (Start-Stop-HPGe) coincidence matrix. It is clear from the spectrum that the majority of the $\gamma$ radiations observed is the result of decays of excited states in ${ }^{208} \mathrm{~Pb}(Z=82)$ and ${ }^{228} \mathrm{Th}$. The former is the result of the $\beta$-decay of ${ }^{208} \mathrm{Tl}(Z=81)$ as the final stage of the ${ }^{232} \mathrm{Th}$ decay chain, while excited states of ${ }^{228} \mathrm{Th}$ are populated as the result of $\beta$-decay of ${ }^{228} \mathrm{Ac}(Z=89)$. There are a few intense $\gamma$ rays associated with the ${ }^{232} \mathrm{Th}$ decay chain that are not observed in the spectra of Fig. 1 due to the decays having unity multiplicity and, therefore, do not generate a TAC output.

The excited states and $\gamma$-ray transitions of interest in ${ }^{228}$ Th are shown in Figure 2. To measure the lifetime of the $J^{\pi}=1^{-}$state, the time difference between the arrival of $795 \mathrm{keV}$ and $328 \mathrm{keV}$ or $795 \mathrm{keV}$ and $270 \mathrm{keV}$ transition photons (as they populate and depopulate the state, respectively) was measured with the TAC. Corresponding TAC spectra are shown in Figure 3a while a sample of the $\gamma$ rays observed in coincidence with the $795 \mathrm{keV}$ transition is presented in Figure 3c. Peaks corresponding to the $270 \mathrm{keV}$ and $328 \mathrm{keV}$ transitions de- 


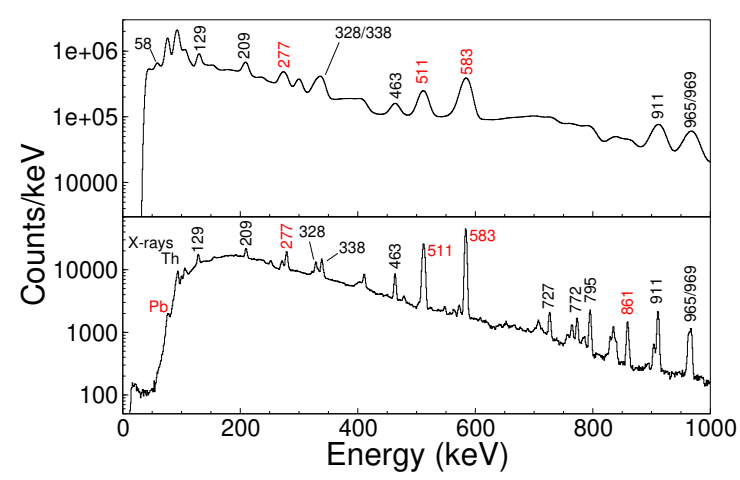

FIG. 1: Gamma-ray spectra resulting from the decay of ${ }^{232} \mathrm{Th}$. The top panel shows $\gamma$ radiations detected with the Start $\mathrm{LaBr}_{3}(\mathrm{Ce})$ detector in coincidence with radiation detected in the Stop $\mathrm{LaBr}_{3}(\mathrm{Ce})$ detector. The bottom panel shows the $\gamma$-ray spectrum detected with a high-purity germanium detector in coincidence with $\gamma$ rays detected in both the Start and Stop detectors. Peaks labelled in black and red are associated with the decay of excited states in ${ }^{228} \mathrm{Th}$ and ${ }^{208} \mathrm{~Pb}$, respectively.

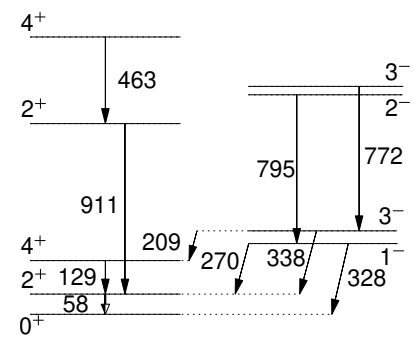

FIG. 2: A partial level scheme showing relevant states and $\gamma$-ray transitions in ${ }^{228} \mathrm{Th}$. The total angular momentum and parity of each state is indicated on the left for states of positive parity and right for negative parity. The $\gamma$-ray transitions are indicated by vertical arrows with the corresponding energy in units of keV shown.

exciting the $1^{-}$state clearly dominate the spectrum of Fig. 3c. In addition, some background peaks corresponding to other intense transitions in the ${ }^{232} \mathrm{Th}$ decay chain can be observed. As a result, the peaks of interest for lifetime measurements sit on top of a background dominated by events corresponding to Compton scattered background $\gamma$ rays. The method outlined by Ansari et al. [29] has been employed to correct for the effect of this background. Following correction, the lifetime of the $J^{\pi}=1^{-}$state was measured to be $\tau=4(3)$ ps with the $795-328 \mathrm{keV}$ combination. Using the $795-270 \mathrm{keV}$ combination it was only possible to measure an upper limit corresponding to $\tau \leq 5 \mathrm{ps}$. This value is consistent with the lifetime measured with the 795-328 keV combination but has likely been affected by the presence of 

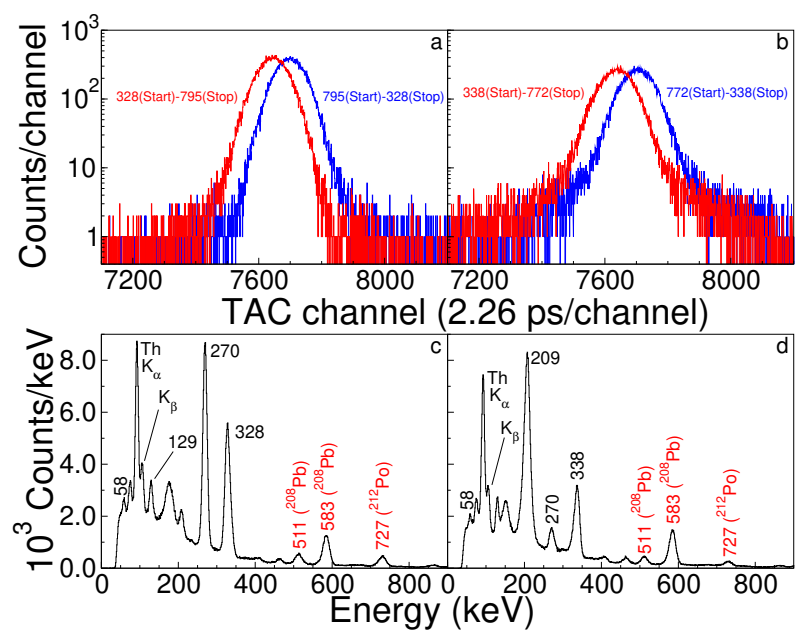

FIG. 3: Timing and $\gamma$-ray energy spectra. a Time-to-amplitude converter (TAC) spectra showing delayed and anti-delayed coincidences between $795 \mathrm{keV}$ and $328 \mathrm{keV}$ transitions populating and depopulating the $J^{\pi}=1^{-}$state; b similar to a but for the $772 \mathrm{keV}$ and $338 \mathrm{keV}$ transitions populating and depopulating the $J^{\pi}=3^{-}$state; c spectrum of $\gamma$ rays in coincidence with $795 \mathrm{keV}$ transition feeding the $J^{\pi}=1^{-}$state; $\mathbf{d} \gamma$ rays in coincidence with $772 \mathrm{keV}$ transitions feeding the $J^{\pi}=3^{-}$state. In panels $\mathbf{c}$ and $\mathbf{d}$, the peaks labelled in black are associated with $\gamma$-ray transitions in ${ }^{228} \mathrm{Th}$.

an intense $277 \mathrm{keV}$ transition in the decay of ${ }^{208} \mathrm{~Pb}$ [30] the influence of which cannot be excluded.

Figure $3 \mathrm{~d}$ shows $\gamma$ radiations observed in coincidence with $772 \mathrm{keV}$ transitions which feed the $J^{\pi}=3^{-}$state. The $209 \mathrm{keV}$ and $338 \mathrm{keV}$ transitions, which have been established to deexcite the $3^{-}$state, dominate this spectrum in addition to some background peaks. The $209 \mathrm{keV}$ peak is significantly wider than that at $338 \mathrm{keV}$ (and indeed the $270 \mathrm{keV}$ peak in Fig. 3c) suggesting the presence of additional background. It is likely that this is due to backscattered photons originating from the other nearby detectors. The TAC spectra corresponding to the $772 \mathrm{keV}$ feeder and the $338 \mathrm{keV}$ decay transitions are shown in Fig. 3b. The background-corrected lifetime for the $3^{-}$state is found to be $\tau=13.2(23)$ ps. In addition to the lifetimes of the low-lying negative parity states, lifetimes of three positive-parity states have been measured and listed in Table I.

The lifetimes of the first $J^{\pi}=2^{+}$and $4^{+}$states have previously been measured using the delayed-coincidence technique, where the difference in time between detection of a populating $\alpha$ particle and the de-exciting $\gamma$-ray was measured [32]. Our results are in good agreement 
TABLE I: Properties of levels and transitions in ${ }^{228} \mathrm{Th}$ as measured in this work. The first three columns list the excitation energies, the total angular momenta and parities and the measured lifetimes. The remaining columns list the reduced transition probabilities, $B(E L)$, and the intrinsic electric dipole, $D_{0}$, and quadrupole moments, $Q_{0}$. To calculate the reduced transition probabilities, data related to the branching ratios, internal coefficients and transition multipolarities have been extracted from Ref. [31].

\begin{tabular}{ccccccc}
\hline \hline $\begin{array}{c}E_{\text {level }} \\
{[\mathrm{keV}]}\end{array}$ & $J_{i}^{\pi} \rightarrow J_{f}^{\pi}$ & $\begin{array}{c}\tau \\
{[\mathrm{ps}]}\end{array}$ & $\begin{array}{c}B(E 1) \\
{\left[10^{-4} \text { W.u. }\right]}\end{array}$ & $\begin{array}{c}\left|D_{0}\right| \\
{[\mathrm{efm}]}\end{array}$ & $\begin{array}{c}B(E 2) \\
{[\text { W.u. }]}\end{array}$ & $\begin{array}{c}\left|Q_{0}\right| \\
{\left[e \mathrm{fm}^{2}\right]}\end{array}$ \\
\hline 58 & $2^{+} \rightarrow 0^{+}$ & $587(12)$ & & & $170(3)$ & $840(17)$ \\
187 & $4^{+} \rightarrow 2^{+}$ & $260(14)$ & & & $224(12)$ & $808(44)$ \\
328 & $1^{-} \rightarrow 0^{+}$ & $4(3)$ & $8(6)$ & $0.16(8)$ & & \\
328 & $1^{-} \rightarrow 2^{+}$ & $\leq 5$ & $\geq 14$ & $\geq 0.14$ & \\
396 & $3^{-} \rightarrow 2^{+}$ & $13.2(23)$ & $3.8(7)$ & $0.09(2)$ & & \\
969 & $2^{+} \rightarrow 2^{+}$ & $3.8(21)$ & & & $2.5(14)$ \\
969 & $2^{+} \rightarrow 0^{+}$ & $3.8(21)$ & & & $1.1(6)$ \\
\hline \hline
\end{tabular}

with these studies confirming a large quadrupole deformation in the ground state. We have also measured the lifetime of the $J^{\pi}=2^{+}$state for an excitation energy of $969 \mathrm{keV}$. Previous studies of the high-spin states of ${ }^{228}$ Th have identified the state as the bandhead of a $K^{\pi}=2^{+}$ $\gamma$-vibrational band [33]. The reduced transition probability, $B\left(E 2 ; 2_{\gamma}^{+} \rightarrow 0^{+}\right)=1.1(6)$ W.u., is similar to the $B\left(E 2 ; 2_{\gamma}^{+} \rightarrow 0^{+}\right)=1.3(5)$ W.u. value measured by Gaffney et al. [25] for the $\gamma$-vibrational bandhead in ${ }^{224} \mathrm{Ra}$. In addition, the $\frac{B\left(E 2 ; 2_{\gamma}^{+} \rightarrow 0^{+}\right)}{B\left(E 2 ; 2_{\gamma}^{+} \rightarrow 2^{+}\right)}=0.45(29)$ ratio is consistent, within uncertainties, with the limit of 0.7 expected from the Alaga rules [34], which further supports the assignment of this level as the $\gamma$-vibrational bandhead in ${ }^{228} \mathrm{Th}$.

The measured lifetime of the $J^{\pi}=1^{-}$state allows for a $B\left(E 1 ; 1^{-} \rightarrow 0^{+}\right)=0.8(6) \times$ $10^{-3}$ W.u. value to be extracted. A $B(E 1)$ rate of this magnitude is consistent with enhanced octupole collectivity, but is similar to the $B\left(E 1 ; 1^{-} \rightarrow 0^{+}\right)<1.5 \times 10^{-3} \mathrm{~W} . \mathrm{u}$. value reported by Gaffney et al. [25] for the octupole-vibrating nucleus ${ }_{86}^{220} \mathrm{Rn}$. Few theoretical predictions exist for the $E 1$ strength of low-energy states of the thorium nucleus. A comprehensive study of the dipole strength in actinide nuclei, performed by Butler and Nazarewicz [3], predicts the $E 1$ strength only for the high-spin states of ${ }^{228} \mathrm{Th}$. A more recent study, performed by 
Robledo and Butler [24], considered the coupling of the quadrupole and octupole collective degrees of freedom to predict $B\left(E 1 ; 1^{-} \rightarrow 0^{+}\right)=1.2 \times 10^{-3}$ W.u. for ${ }^{228} \mathrm{Th}$.

The intrinsic dipole moment, $D_{0}$, can be calculated directly from the measured $B(E 1)$ rates according to the rotational model formula:

$$
B\left(E 1 ; J_{i} \rightarrow J_{f}\right)=\frac{3}{4 \pi} D_{0}^{2}\left\langle J_{i} K_{i} 10 \mid J_{f} K_{f}\right\rangle^{2}
$$

where $\left\langle J_{i} K_{i} 10 \mid J_{f} K_{f}\right\rangle$ is the Clebsch-Gordan coefficient. The use of a rotational model formula is appropriate for a nucleus such as ${ }^{228} \mathrm{Th}$, which exhibits clear signs of rotational motion $\left(Q_{0}=840(17) \mathrm{efm}^{2}\right.$ and $\left.E\left(4_{1}^{+}\right) / E\left(2_{1}^{+}\right)=3.22\right)$. This formula is commonly applied to the actinide region, regardless of how rotational the nuclei appear, to obtain a consistent measure of the dipole moment from available data. Our measurement results in a value of $D_{0}=0.16(8)$ efm for the $J^{\pi}=1^{-}$state. This $D_{0}$ value, while consistent within one standard deviation, is larger than the values reported for any of the higher spin states of ${ }^{228}$ Th extracted indirectly from observed $B(E 1) / B(E 2)$ ratios [35, 36]. This dipole moment is also larger than the prediction of $D_{0}=0.07$ efm for $J^{\pi}=8^{-}$by Butler and Nazarewicz [3] indicating that the dipole moment, and possibly, the octupole collectivity in this nucleus was underestimated in their calculations.

The intrinsic dipole moment of $D_{0}=0.09(2)$ efm calculated in the current work for the $J^{\pi}=3^{-}$state in ${ }^{228} \mathrm{Th}$ is consistent with the results of Ackermann et al. [36] in which a lower limit $\left(D_{0} \geq 0.1 \mathrm{efm}\right)$ was determined by means of $B(E 1) / B(E 2)$ ratios. The lower limit in the previous study corresponds to a maximum value of the observed branching of the $3^{-} \rightarrow 1^{-} E 2$ transition. It is worth noting that the value of $D_{0}$ measured for the $J^{\pi}=3^{-}$ state in our work is in good agreement with the values established for higher-spin states in the octupole band of ${ }^{228}$ Th which range from $0.11-0.13[35,36]$.

The fact that the calculations of Robledo and Butler [24] reproduce the experimentallydetermined $B\left(E 1 ; 1^{-} \rightarrow 0^{+}\right)$value suggests that they can be used to estimate the extent of octupole collectivity in the low-energy states of ${ }^{228} \mathrm{Th}$. In the case of the ground state of ${ }^{228} \mathrm{Th}$, the calculations of Ref. [24] predict a pronounced minimum in the potential energy corresponding to an octupole moment $Q_{3}=3500 \mathrm{efm}^{3}$. These calculations overestimate the octupole moment in ${ }^{224} \mathrm{Ra}$, which was measured to be $Q_{3}=2520(90) \mathrm{efm}^{3}$ [25], by a factor of $\sim 15 \%$. Scaling the octupole moment for ${ }^{228} \mathrm{Th}$ to $2950 \mathrm{efm}^{3}$ and using the measured quadrupole moment $Q_{0}\left(2^{+}\right)=840(17) \mathrm{efm}^{2}$, the equations described by Leander and 
Chen [37] have been used (neglecting terms higher than $L=3$ ) to calculate the quadrupole and octupole deformation parameters $\beta_{2}=0.21(1)$ and $\beta_{3}=0.11(2)$ for ${ }^{228} \mathrm{Th}$. The uncertainties reported here include the effects of measurements and the scaling factor in the octupole moment. These values of the deformation parameters are comparable with the theoretical values of $\beta_{2}=0.21$ and $\beta_{3}=0.15$ reported by Agbemava et al. [9]. Dobaczewski et al. [38] have predicted that the Schiff moments of odd- $A$ actinide nuclei are correlated with the octupole moments in the neighbouring even-even nuclei. Comparing the octupole deformation obtained as a result of this work with those reported for ${ }^{224} \mathrm{Ra}$ [25] $\left(\beta_{2}=0.154\right.$ and $\left.\beta_{3}=0.097\right)$ indicates that odd- $A$ nuclei composed of a ${ }^{228} \mathrm{Th}$ core, such as ${ }^{229} \mathrm{~Pa}$ and ${ }^{229} \mathrm{Th}$, may be superior candidates for the search for a nuclear EDM. The increased $\beta_{3}$ value for ${ }^{228} \mathrm{Th}$ means that the collective Schiff moment may be larger in ${ }^{229} \mathrm{~Pa}$ and ${ }^{229} \mathrm{Th}$ compared with odd- $A$ Rn and Ra nuclei currently attracting attention in searches for an atomic $\operatorname{EDM}[39,40]$.

Excited states of ${ }^{228} \mathrm{Th}$ are populated by the $\beta$-decay of the ground state of ${ }_{89}^{228} \mathrm{Ac}$ following the $\alpha$-decay of the ${ }^{232} \mathrm{Th}$ nucleus. A foil of natural $99.5 \%$ purity thorium with dimensions of $50 \times 50 \times 0.05 \mathrm{~mm}$ acted as a source of ${ }^{232}$ Th nuclei with an activity corresponding to approximately $6 \mathrm{kBq}$. Due to being in a state of secular equilibrium the foil provided $\sim 6 \mathrm{kBq}$ of ${ }^{228}$ Ac. The thorium foil was located equidistant between two cerium-doped lanthanum bromide $\left(\mathrm{LaBr}_{3}(\mathrm{Ce})\right)$ detectors, the front faces of which were separated by $7 \mathrm{~mm}$. The thorium foil was oriented such that short axis was aligned along the $\mathrm{LaBr}_{3}(\mathrm{Ce})-\mathrm{LaBr}_{3}(\mathrm{Ce})$ detector axis. This arrangement ensured that the difference in distance between a decaying nucleus and each of the $\mathrm{LaBr}_{3}(\mathrm{Ce})$ detectors remains less than $0.05 \mathrm{~mm}$ at all times. This ensured that any offsets in the time spectra were reduced to below $0.2 \mathrm{ps}$. The cylindrical $\mathrm{LaBr}_{3}(\mathrm{Ce})$ crystals each had a diameter and length of $25.4 \mathrm{~mm}$ and are coupled to Hamamatsu R9420 photomultiplier tubes. Each detector was located $3.5 \mathrm{~mm}$ from the thorium foil. An Ortec GEM P-type high-purity germanium (HPGe) detector was orientated $90^{\circ}$ relative to the $\mathrm{LaBr}_{3}(\mathrm{Ce})-\mathrm{LaBr}_{3}(\mathrm{Ce})$ detector axis. This detector had a relative efficiency of $59 \%$ and an endcap diameter of $70 \mathrm{~mm}$. Signals from the $\mathrm{LaBr}_{3}(\mathrm{Ce})$ detectors were optimised for timing measurements and connected to a time-to-amplitude converter (TAC), which produced an output voltage proportional to the difference in time between detection of two detected $\gamma$-ray photons, respectively.

Waveform traces from each of the detectors and the TAC were recorded using a CAEN 


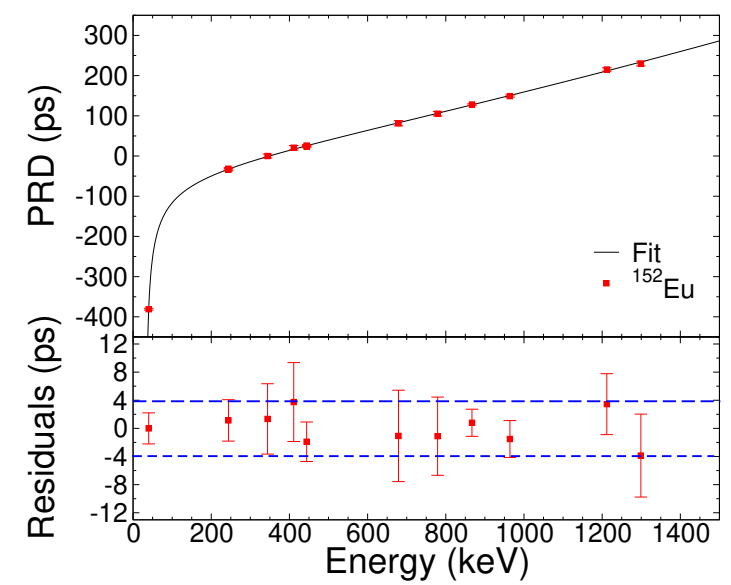

FIG. 4: Top shows the $\gamma-\gamma$ time-walk dependence with $\gamma$-ray energy of the fast-timing apparatus.

Data points correspond to transitions depopulating prompt states in ${ }^{152} \mathrm{Sm}$ and ${ }^{152} \mathrm{Gd}$ following the $\beta$-decay of ${ }^{152} \mathrm{Eu}$. Error bars are smaller than the symbols for the data points. Bottom shows the differences between the data and the fitted prompt response function with the dashed line indicating two standard deviations.

V1725 digitiser and stored using the MIDAS data acquisition software [41]. Custom software was used to reduce the data by extracting the pulse heights from the traces using a moving window deconvolution algorithm. The reduced data were subsequently analysed using the Root data analysis framework [42].

The lifetimes of excited states were measured according to the mirror symmetric centroid difference (MSCD) method outlined by Regis et al. [43]. This method involves two $\gamma$-ray detectors, the signals of which are processed by a constant-fraction discriminator (CFD). The resultant logic signal from one CFD is used as the Start input of the TAC while the other is artificially delayed before providing the Stop signal for the TAC. The method involves using the Start detector to detect the $\gamma$-ray photons populating an excited state of interest and the Stop detector to detect $\gamma$ rays depopulating the state. As a result, a spectrum of TAC values with a Gaussian distribution is obtained when the lifetime of the state is sufficiently short $(\tau \leq 150 \mathrm{ps})$. For longer lifetimes the Gaussian distribution has an exponential tail. This configuration is known as the delayed configuration. In the anti-delayed configuration the Start detector is used to detect the depopulating transition while the Stop detects the populating transition. The difference between the first moments of the delayed and anti-delayed TAC spectra $(\Delta C)$ is proportional to the lifetime of the state. However, a natural 'time-walk' is associated with the CFD, which must be accounted for to obtain 
accurate lifetimes. The effect of the time-walk is such that $\Delta C$ values will increase as the energy of the detected transitions increases regardless of the lifetime of the state. The time-walk can be quantified by measuring $\Delta C$ as a function of energy for precisely known picosecond transitions. There are a number of suitable states in the ${ }^{152} \mathrm{Sm}$ and ${ }^{152} \mathrm{Gd}$ nuclei that are populated as a result of the $\beta$-decay of a standard ${ }^{152} \mathrm{Eu} \gamma$-ray calibration source. The resulting $\Delta C$ values obtained using the prompt states in ${ }^{152} \mathrm{Sm}$ and ${ }^{152} \mathrm{Gd}$ are shown in Extended Data Figure 1 as a function of the energy of the depopulating transition. The resulting curve fitted to the ${ }^{152} \mathrm{Eu}$ data is known as the prompt response function (typically referred to as PRD) of the fast-timing apparatus, which is used to correct for the CFD time-walk. The lifetime is calculated as $\tau=(\Delta C-\triangle P R D) / 2$, where $\triangle P R D$ is the difference between PRD values for the $\gamma$-ray energies detected with the Start and Stop detectors, respectively. To check that the PRD curve obtained with the ${ }^{152} \mathrm{Eu}$ calibration source remained valid for the thorium foil, measurements of lifetimes of excited states in other nuclei, also populated in the decay of ${ }^{232} \mathrm{Th}$, have been performed and compared with literature values. For example, the first excited state in ${ }^{212} \mathrm{Po}$ was measured to have a lifetime of $19.5(25)$ ps which agrees with the value of 20.5(26) ps reported by Kocheva et al. [44]. In addition, the lifetime of the second excited state $\left(E_{\text {level }}=3198 \mathrm{keV} ; J^{\pi}=5^{-}\right)$in ${ }^{208} \mathrm{~Pb}$ was measured as $443(2) \mathrm{ps}$ in good agreement with the error-weighted literature value of $424(22)$ ps [30].

\section{Data availability}

Source data are available for this paper. All other data that support the plots within this paper and other findings of this study are available from the corresponding author upon reasonable request.

\section{Code availability}

The codes used to process the data presented in this paper are available from the corresponding author upon reasonable request. 


\section{Acknowledgments}

The authors would like to acknowledge useful discussions with Luis Robledo who was the recipient of a SUPA Distinguished Visitor grant awarded to UWS. Financial support for this work has been provided by the Scottish Funding Council (SFC) and the UK Science and Technology Facilities Council (STFC). We acknowledge support of the U.K. EPSRC (Grant No. EP/J018171/1, EP/J500094/1 and EP/N028694/1), the ECs LASERLAB-EUROPE (Grant No. 654148), EuCARD-2 (Grant No. 312453), EuPRAXIA (Grant No. 653782), ARIES (Grant No. 730871) and the Extreme Light Infrastructure (ELI) European Project.

\section{Author Contributions}

D.O'D. conceived the investigation; M.M.C., D.O'D. and G.B. set up the instrumentation; M.M.C., D.O'D., G.B. and P.S. performed the data analysis; M.M.C., D.O'D. and M.S. interpreted the results; D.O'D., M.B., D.A.J., B.S.N.S., M.S., P.S. and J.F.S. contributed to writing the manuscript.

\section{Additional Information}

Competing Interests: The authors declare no competing interests.

[1] Bohr, A. The coupling of nuclear surface oscillations to the motion of individual nucleons. Dan. Mat. Fys. Medd. 26, No. 14 (1952).

[2] Bohr, A. \& Mottelson, B. R. Collective and individual-particle aspects of nuclear structure. Dan. Mat. Fys. Medd. 27, No. 16 (1953).

[3] Butler, P. A. \& Nazarewicz, W. Intrinsic dipole moments in reflection-asymmetric nuclei. Nucl. Phys. A 533, 249-268 (1991).

[4] Regan, B. C., Commins, E. D., Schmidt, C. J. \& Demille, D. New Limit on the Electron Electric Dipole Moment. Phys. Rev. Lett. 88, 071805 (2002).

[5] Hudson, J. J. et al. Improved measurement of the shape of the electron. Nature 473, 493-496 (2011). 
[6] Baron, J. et al. Order of Magnitude Smaller Limit on the Electric Dipole Moment of the Electron. Science 343, 269-272 (2014).

[7] Cairncross, W. B. et al. Precision Measurement of the Electron's Electric Dipole Moment Using Trapped Molecular Ions. Phys. Rev. Lett. 119, 153001 (2017).

[8] ACME Collaboration. Improved limit on the electric dipole moment of the electron. Nature 562, 355-360 (2018).

[9] Agbemava, S. E., Afanasjev, A. V. \& Ring, P. Octupole deformation in the ground states of even-even nuclei: A global analysis within the covariant density functional theory. Phys. Rev. C 93, 044304 (2016).

[10] Schiff, L. I. Measurability of Nuclear Electric Dipole Moments. Phys. Rev. 132, 2194-2200 (1963).

[11] Sushkov, O., Flambaum, V. \& Khriplovich, I. Possibility of investigating p-and t-odd nuclear forces in atomic and molecular experiments. Zh. Eksp. Teor. Fiz 87, 1521-1540 (1984).

[12] Flambaum, V. V., Khriplovich, I. B. \& Sushkov, O. P. Limit on the constant of Tnonconserving nucleon-nucleon interaction. Physics Letters B 162, 213-216 (1985).

[13] Auerbach, N., Flambaum, V. V. \& Spevak, V. Collective t- and p-odd electromagnetic moments in nuclei with octupole deformations. Phys. Rev. Lett. 76, 4316-4319 (1996). URL https://link.aps.org/doi/10.1103/PhysRevLett.76.4316.

[14] Spevak, V., Auerbach, N. \& Flambaum, V. V. Enhanced T-odd, P-odd electromagnetic moments in reflection asymmetric nuclei. Phys. Rev. C 56, 1357-1369 (1997).

[15] Ellis, J., Lee, J. S. \& Pilaftsis, A. Maximal electric dipole moments of nuclei with enhanced schiff moments. Journal of High Energy Physics 2011, 45 (2011).

[16] Chasman, R. R. Incipient octupole deformation and parity doublets in the odd mass light actinides. Phys. Lett. B 96, 7-10 (1980).

[17] Ahmad, I., Gindler, J. E., Betts, R. R., Chasman, R. R. \& Friedman, A. M. Possible GroundState Octupole Deformation in ${ }^{229} \mathrm{~Pa}$. Phys. Rev. Lett. 49, 1758-1761 (1982).

[18] Grafen, V. et al. Does a $5 / 2^{+}-5 / 2^{-}$ground-state parity doublet exist in ${ }^{229} \mathrm{~Pa}$. Phys. Rev. C 44, R1728-R1731 (1991).

[19] Lösch, A. et al. The low-lying levels in ${ }^{229} \mathrm{~Pa}$ and parity doublets. Z. Phys. A 348, 235-235 (1994).

[20] Ahmad, I., Chasman, R. R., Greene, J. P., Kondev, F. G. \& Zhu, S. Electron capture decay 
of 58-min ${ }_{92}^{229} \mathrm{U}$ and levels in ${ }_{91}^{229} \mathrm{~Pa}$. Phys. Rev. C 92, 024313 (2015).

[21] Flambaum, V. V. Enhanced nuclear Schiff moment and time-reversal violation in ${ }^{229}$ Thcontaining molecules. Phys. Rev. C 99, 035501 (2019).

[22] Stephens, F., Asaro, F. \& Perlman, I. Low-lying 1- states in even-even nuclei. Phys. Rev. 96, 1568-1572 (1954).

[23] Stephens, F. S., Asaro, F. \& Perlman, I. Radiations from $1^{-}$states in even-even nuclei. Phys. Rev. 100, 1543-1545 (1955).

[24] Robledo, L. M. \& Butler, P. A. Quadrupole-octupole coupling in the light actinides. Phys. Rev. C 88, 051302 (2013).

[25] Gaffney, L. P. et al. Studies of pear-shaped nuclei using accelerated radioactive beams. Nature 497, 199-204 (2013).

[26] Wollersheim, H. J. et al. Coulomb excitation of ${ }^{226}$ Ra. Nucl. Phys. A 556, 261-280 (1993).

[27] Egido, J. L. \& Robledo, L. M. Microscopic study of the octupole degree of freedom in the radium and thorium isotopes with gogny forces. Nucl. Phys. A494, 85-101 (1989).

[28] Butler, P. A. \& Nazarewicz, W. Intrinsic reflection asymmetry in atomic nuclei. Rev. Mod. Phys. 68, 349-421 (1996).

[29] Ansari, S. et al. Experimental study of the lifetime and phase transition in neutron-rich 98,100,102Zr. Phys. Rev. C 96, 054323 (2017).

[30] Martin, M. Nuclear data sheets for a = 208. Nuclear Data Sheets 108, 1583 - 1806 (2007).

[31] Abusaleem, K. Nuclear data sheets for a=228. Nuclear Data Sheets 116, 163 - 262 (2014).

[32] Ton, H., Beens, W., Roodbergen, S. \& Blok, J. Lifetimes of $2^{+}$and $4^{+}$rotational states in heavy doubly even nuclei. Nucl. Phys. A155, 235-244 (1970).

[33] Weber, T., Gröger, J., Günther, C. \& deBoer, J. Study of the first-excited $K^{\pi}=0^{+}$and $2^{+}$ bands in ${ }^{228} \mathrm{Th}$ in the ${ }^{226} \mathrm{Ra}(\alpha, 2 \mathrm{n})$ reaction. Eur. Phys. J. A 1, 39-44 (1998).

[34] Alaga, G., Alder, K., Bohr, A. \& Mottelson, B. R. . Dan. Mat. Fys. Medd. 29, No. 9 (1955).

[35] Schüler, P. et al. High-spin states in ${ }^{224,226,228} \mathrm{Th}$ and the systematics of octupole effects in even Th isotopes. Phys. Lett. B 174, 241-245 (1986).

[36] Ackermann, B. et al. Collective E1 transitions in even-A Ra, Th, and U nuclei. Nucl. Phys. A559, 61-82 (1993).

[37] Leander, G. A. \& Chen, Y. S. Reflection-asymmetric rotor model of odd $A \sim 219-229$ nuclei. Phys. Rev. C 37, 2744-2778 (1988). 
[38] Dobaczewski, J., Engel, J., Kortelainen, M. \& Becker, P. Correlating Schiff Moments in the Light Actinides with Octupole Moments. Phys. Rev. Lett. 121, 232501 (2018). 1807.09581.

[39] Parker, R. H. et al. First Measurement of the Atomic Electric Dipole Moment of ${ }^{225}$ Ra. Phys. Rev. Lett. 114, 233002 (2015).

[40] Bishof, M. et al. Improved limit on the ${ }^{225}$ Ra electric dipole moment. Phys. Rev. C 94, 025501 (2016).

[41] Pucknell, V. \& Unsworth, C. Multi instance data acquisition system. http://npg.dl.ac.uk/MIDAS. Accessed: 15/12/2019.

[42] Brun, R. \& Rademakers, F. ROOT - An object oriented data analysis framework. Nucl. Instr. Meth. Phys. Res. A 389, 81-86 (1997).

[43] Régis, J. M., Pascovici, G., Jolie, J. \& Rudigier, M. The mirror symmetric centroid difference method for picosecond lifetime measurements via $\gamma-\gamma$ coincidences using very fast $\mathrm{LaBr}{ }_{3}(\mathrm{Ce})$ scintillator detectors. Nucl. Instr. Meth. Phys. Res. A 622, 83-92 (2010).

[44] Kocheva, D. et al. Low collectivity of the $2_{1}^{+}$state of ${ }^{212}$ Po. Phys. Rev. C 96, 044305 (2017). URL https://link.aps.org/doi/10.1103/PhysRevC.96.044305. 